\title{
Estrategias para la biopsia de próstata. Revisión de la literatura
}

\author{
Ramírez Backhaus M, Trassierra Villa M, Arlandis Guzmán S, Delgado Oliva F, \\ Boronat Tormo F, Jiménez Cruz JF. \\ Servicio de Urología. Hospital Universitario La Fe. Valencia. \\ Actas Urol Esp. 2007;31(10):1089-1099
}

\section{RESUMEN}

ESTRATEGIAS PARA LA BIOPSIA DE PRÓSTATA. REVISIÓN DE LA LITERATURA

Introducción: Desde que en 1987 se describió la ecografía transrectal como técnica para dirigir la biopsia de próstata, diferentes modalidades de la misma han sido utilizadas para la detección del cáncer de próstata.

Material y Método: Realizamos una revisión de las diferentes técnicas y esquemas de distribución de los cilindros de la biopsia prostática, así mismo describimos la preparación del paciente y las complicaciones más relevantes.

Resultados: La mayoría de los trabajos al respecto comunica una mejora de la sensibilidad de la prueba con las biopsias prostáticas transrectales ecodirigidas ampliadas respecto a la técnica sextante clásica. Dicha ampliación se da a expensas de cilindros generalmente de las zonas más laterales de la zona periférica.

Conclusiones: Hasta el momento, dado que se cuenta con una amplia experiencia y la tasa de complicaciones es asumible, la biopsia prostática transrectal ecodirigida, debiera ser ampliada respecto a la sextante clásica a expensas de cilindros de las zonas periféricas laterales prostáticas.

Palabras clave: Próstata. Biopsia. Cáncer de próstata.

\section{ABSTRACT \\ PROSTATE BIOPSY STRATEGIES. A REVIEW OF THE LITERATURE}

Introduction: In 1987 transrectal ultrasound was described like the technique for guiding prostate biopsy. Since that time different options of transrectal ultrasound guided prostate biopsy were described.

Material and Methods: We did a reviewed of the different techniques and cores distribution in the prostate biopsy, also we describes the patient preparation and the most important complications.

Results: The majority of the reviewed showed an increase in the sensibility rates with the extended transrectal ultrasound guided prostate biopsies. These improvements generally are due to the most lateral zones.

Conclusion: Until now, due to a great experience and a low complications rate, the transrectal ultrasound guided prostate biopsy strategy should be extended respect the classical sextant biopsy with cores from the most lateral zones of the prostate.

Keywords: Prostate. Biopsy. Prostate cancer.

$\mathrm{C}$ omo ocurre en la mayoría de las ocasiones el desarrollo tecnológico militar precede y se traslada a la medicina ${ }^{1}$. La ecografía no se aplica al mundo sanitario hasta después de la segunda guerra mundial. En el campo de la Urología lo utiliza por primera vez Schlegel en 1961 para la valoración de los cálculos renales ${ }^{2}$ y Watanabe et al. en el 67 describieron la primera ecografia transrectal (ETR) ${ }^{3}$. En los años 80 con el creciente interés por el cáncer de próstata y la determi- 
nación del antígeno prostático específico (PSA) ${ }^{4}$ como marcador tumoral, la ecografía transrectal se convierte en una técnica imprescindible para la biopsia de próstata. Se desarrollan por tanto con mucha rapidez los transductores de alta frecuencia que permiten identificar determinadas características del cáncer de próstata ${ }^{4}$ y los dispositivos de resorte que facilitan la obtención del tejido prostático ${ }^{5}$.

En su comienzo la biopsia de próstata se realizaba a ciegas sobre las zonas sospechosas según el tacto rectal (TR). En 1989 Torp-Pedersen et al. ${ }^{6}$ introducen la biopsia de próstata ecodirigida como técnica diagnóstica en el cáncer de próstata. Aparecen los primeros estudios que demostraban la superioridad de la biopsia ecodirigida frente a la digitodirigida ${ }^{7}$, ya que aumentaba la sensibilidad y facilitaba la punción de las zonas sospechosas. Más tarde y con el fin de encontrar aquellos tumores clínicamente silentes surge el método de la biopsia en sextante, base $u$ origen de casi todas las nuevas modalidades de muestreo prostático.

Las limitaciones de la ETR para detectar el cáncer de próstata consisten en que la mayoría de las lesiones hipoecóicas no son cánceres y el $50 \%$ de los cánceres no palpables con un diámetro menor de $1 \mathrm{~cm}$ no se observan en la ecografia ${ }^{8}$. Si bien las áreas hipoecóicas de los ultrasonidos tienen 2 veces más probabilidades de ser un cáncer que las isoecoicas ${ }^{9-11}$, entre el 25 y el $50 \%$ de los cánceres pasaría inadvertido si únicamente se biopsiaran las áreas hipoecoicas ${ }^{12}$. En los últimos años y desde la aparición del PSA son escasas las referencias en la bibliografia acerca de las características sonográficas de tumores prostáti$\cos ^{12}$ y actualmente se están investigando la utilidad de algunos contrastes sonográficos o ecografias tridimensionales con el fin de aumentar la sensibilidad en la detección del cáncer de próstata $^{13-17}$. Por el momento debe de quedar claro que la ETR es una técnica que asegura la biopsia precisa dentro un área amplia de tejido prostático ${ }^{18}$.

\section{TÉCNICA}

La preparación de los pacientes a los que se va a realizar una biopsia de próstata transrectal consta de tres partes fundamentales: Preparación del recto, profilaxis antibiótica y analgesia.

\section{Preparación rectal}

Pese a lo que a priori pueda parecer, son muchos los trabajos que abogan por no limpiar el recto para la biopsia de próstata. Vallancien et al. ${ }^{19}$ objetivaron un aumento de las complicaciones infecciosas en los pacientes sometidos a enemas de limpieza y postulaban que la irritación rectal producida por el lavado facilitaría la bacteriemia. Por otro lado, un pequeño estudio prospectivo de Brown et al. ${ }^{20}$ objetivó una menor tasa de pacientes con infección del tracto urinario y fiebre en los pacientes a los que pautaba enemas con povidonaiodada. Lindert et al. sí que observaron mayor proporción de bacteriemias en pacientes sin limpieza del recto, si bien éstos no llevaban profilaxis antibiótica y las bacteriemias fueron asintomáticas ${ }^{21}$.

En un estudio realizado por $\mathrm{Carey}^{22}$ con 448 pacientes sometidos a biopsia transrectal bajo profilaxis antibiótica con ciprofloxacino $500 \mathrm{mg}$ la noche antes de la prueba y un comprimido cada 12 horas durante los tres días que siguen a la intervención, no se observaron diferencias en cuanto a complicaciones infecciosas e inflamatorias en los pacientes a los que se aplicaba enemas y los que no se les preparaba el recto ${ }^{22}$.

De hecho en una encuesta realizada por Davis et al. ${ }^{23}$ a 110 urólogos norteamericanos objetivan que el $79 \%$ de ellos aplican algún tipo de enema rectal, la mayoría de ellos inmediatamente antes de la intervención, sólo un 7\% lo pautan la noche de antes e inmediatamente antes de la prueba, mientras un $14 \%$ no preparan el recto. Cuando estratifican en función del centro, observan que un $27 \%$ de los centros universitarios no pautan enemas de limpieza ${ }^{23}$.

Por tanto no existe en la práctica clínica habitual, ni en la literatura, consenso o protocolo alguno en este tema. En nuestro centro aconsejamos al paciente la aplicación de un enema de limpieza la noche antes de la biopsia y otro la mañana de la prueba.

\section{Control del dolor operatorio}

La ecografia transrectal y biopsia de próstata es una procedimiento incómodo. La preocupación sobre la posibilidad de tener un cáncer, la vía de acceso anal, el hecho de que la próstata sea un órgano del aparato genital masculino y la 
propia lectura del consentimiento informado generan en el paciente una sensación de estrés, inquietud y nerviosismo; y hace que conciban la biopsia de próstata como un procedimiento traumático. De un $7 \%{ }^{24}$ a un $90 \%{ }^{25}$ de los pacientes refieren sensación molesta. Probablemente los pacientes más ansiosos refieran más dolor o molestia durante la prueba.

Hay varias técnicas descritas en la literatura para el adecuado control del dolor durante la biopsia. Se trata de métodos anestésicos locales o tópicos, sedación o analgesia.

\section{Anestesia local}

Los pioneros en la utilización de la anestesia para el bloqueo de los plexos nerviosos periprostáticos fueron Nash et al. ${ }^{26}$ en 1996. La mayoría de los procedimientos anestésicos consisten en la inyección periprostática en distintas zonas de lidocaína al 1\%. Hay autores que realizan las inyecciones en las regiones periféricas craneal, medial y apical ${ }^{27}$. Otros proponen la inyección en el ángulo prostatoseminal ${ }^{28}$. También está descrita la introducción de $10 \mathrm{ml}$ entre las fascias de Denonvilliers y la periprostática ${ }^{29}$ o a ambos lados del ápex prostático ${ }^{30}$. La bupivacaína $0,25 \%$, lidocaína $2 \%$ o articaína al $1 \%$ son otros anestésicos utilizados. También la lidocaína $2 \%$ en gel como anestésico tópico 10 minutos antes de la anestesia, descrita por Isa et al. ${ }^{31}$ Existen estudios aleatorizados que demuestran mayor efectividad del bloqueo nervioso que la aplicación de anestesia tópica ${ }^{32}$, sobre todo cuando se trata de biopsias ampliadas, sin embargo no está exento de complicaciones ya que probablemente aumente el riesgo de sangrado y de infecciones.

\section{Sedación}

Se han utilizado fármacos con un periodo de inducción muy corto, fácilmente reversible y con escasos efectos secundarios. Masood et al. ${ }^{33}$ realizaron un estudio aleatorizado a doble ciego con inhalación de entonox, gas anestésico de uso generalizado en el Reino Unido. También está descrito el uso de propofol ${ }^{34}$ con resultados excelentes aunque se necesita un anestesiólogo con el consiguiente incremento del gasto.

\section{Analgésicos}

El rofecoxib oral no ha demostrado diferencias con el placebo en un trabajo realizado por Moinzadeh et al. ${ }^{35}$, Haq et al. ${ }^{36}$ concluyen en un trabajo con diclofenaco en supositorios que mejora la tolerancia de la prueba sin aumentar la morbilidad. La petidina a dosis de $1 \mathrm{mg} / \mathrm{kg}$ diluida en $100 \mathrm{ml}$ de suero es otra de las opciones descritas.

En el estudio de Davis et al. ${ }^{23}$ el 33\% de los urólogos norteamericanos no administran analgesia alguna a los pacientes biopsiados, un 18\% y un $16 \%$ pautan anestesia local (gel o supositorios) y analgesia respectivamente. El $23 \%$ de los urólogos de centros universitarios realizan un bloqueo de los plexos periprostáticos, mientras el porcentaje de médicos que combinan los tratamientos es ínfimo.

Probablemente se deberían identificar los pacientes que se beneficiarian en mayor medida de la anestesia. Según Bastide et al. ${ }^{37}$ se trataría de pacientes jóvenes, ansiosos a los que se les va a realizar una biopsia ampliada o de repetición.

\section{Profilaxis antibiótica}

En la literatura norteamericana y anglosajona coinciden en que un régimen de fluorquinolonas oral sería suficiente ${ }^{38}$. En nuestro medio debido a la alta resistencia a fluorqinolonas la terapia ideal es controvertida. En nuestro centro utilizábamos una pauta consistente en un aminoglucósido (tobramicina $100 \mathrm{mg}$ endovenosa) media hora antes de la biopsia y otra dosis intramuscular ambulatoria aproximadamente a las doce horas de la primera dosis. Vimos un incremento de las complicaciones infecciosas, constatando cuatro casos de sepsis por prostatitis aguda en un corto periodo de tiempo, lo que motivó replantearnos la estrategia profiláctica. Se realizó un estudio prospectivo con 153 pacientes comparando nuestra pauta clásica frente otro grupo al que añadiamos ciprofloxacino 500mg oral cada 12 horas, durante 72 horas tras la biopsia. Observamos una menor tasa de complicaciones infecciosas estadísticamente significativa, en el grupo de la nueva estrategia profiláctica ${ }^{39}$.

En la encuesta de Davis et al. ${ }^{23}$ el $81 \%$ de los facultativos encuestados sólo administra una fluorquinolona oral antes del procedimiento, mien- 
tras que fluorquinolona más aminoglucósido es la posología elegida por el $17 \%$. El 56\% comenzaban el tratamiento el día previo a la biopsia y un $16 \%$ continuaban con el tratamiento oral 24-48 horas post biopsia, el $4 \%$ de los encuestados utilizaba el régimen que empleamos en nuestro centro; aminoglucósido parenteral pre-biopsia y una pauta de tres días de quinolona oral.

La duración del tratamiento oral después de la biopsia también es objeto de controversia y hay estudios que sostienen que la duración de tres o cuatro días de tratamiento oral disminuye de forma significativa la tasa de complicaciones infecciosas ${ }^{40-42}$.

\section{ESQUEMAS DE PUNCIÓN PROSTÁTICA (Fig. 1)}

Biopsia en sextante

Introducida por Hodge $^{7}$ en 1989, ha sido durante mucho tiempo el patrón de referencia ("gold standard") de las técnicas de biopsia. Se obtienen cilindros de las zonas craneal, medial y apical parauretrales (en un punto intermedio entre la línea media y el borde lateral de la próstata, en un corte transversal) de ambos lóbulos ${ }^{7}$. Para disminuir el número de falsos negativos dicha técnica se ha modificado desplazando las punciones más lateralmente, sin aumentar el número de cilíndros ${ }^{12,43}$. Rodríguez-Patrón et al. ${ }^{12}$ en la serie más importante publicada en nuestro país, utilizan esta técnica en 7127 biopsias con una tasa diagnóstica de cáncer de 42,6\% cuando se trataba de primera indicación si bien la tasa se reduce al 29,6\% cuando se trata de pacientes con un PSA entre 4 y 10.

\section{Biopsia de próstata de las cinco regiones}

Con el objetivo de ampliar la toma de muestras y poder examinar con más detalle toda la zona periférica de la glándula surge esta modalidad, que propone la obtención de los cilindros de la técnica clásica a los que añade dos de la zona más lateral de cada lóbulo prostático así como tres cilindros de la zona medial ${ }^{44,45}$ de modo que se obtienen un total de 13 cilindros. Además se propone aumentar un cilindro más de cada zona si el volumen prostático excede de los 50 gramos. Eskew et al. fueron los primeros en diseñar un estudio prospectivo donde comparaban esta técnica con la sextante, objetivando una reducción del $35 \%$ en los falsos negativos; un $88 \%$ de los tumores que se diagnostican, excluidos los de la sextante, son localizados en las zonas más laterales (cuernos prostáticos laterales) ${ }^{46}$.

\section{Sumatorio de dos biopsias en sextante}

Esta estrategia propuesta por Levine et al. ${ }^{47}$ consiste en realizar en el mismo acto la toma de doce cilindros con la misma disposición de la técnica clásica. Se examinaron las biopsias de 137 pacientes y se observó un $28 \%$ de tumores y pin adicionales $^{45}$.

Biopsia con toma de 11 cilindros Babaian et al. hacen un estudio con una estrategia que consta de una distribución de cilindros en sextante mas la toma de muestras de las dos zonas transicionales, otra de los cuernos laterales de ambos lóbulos y una de la zona más medial ${ }^{45}$. Con esta técnica los autores no encuentran un aumento significativo de la tasa de detección de cáncer, aunque sí que encuentran mayor rentabilidad diagnóstica para segundas biopsias en pacientes con sospecha de adenocarcinoma con biopsia previa negativa. 


\section{Biopsia de ocho cilindros}

Dicha estrategia surge del estudio de Presti et al. ${ }^{44}$ que biopsian a 483 pacientes con toma de 10 cilindros a cada uno (la sextante mas dos cilindros de la zona más lateral de cada lado). Con dicha distribución los autores consideran que pueden detectar un 96\% de los tumores, además si retiran los cilindros de la zona periférica craneal de la sextante de ambos lóbulos sólo dejan de diagnosticar un $1 \%$ de los tumores ${ }^{44}$. Esta técnica es la misma que la de las cinco regiones eliminando las biopsias mediales, es decir, una técnica en sextante a la que añadimos dos biopsias de los cuernos lateroprostáticos de cada lóbulo, y se elimina la biopsia de la zona periférica craneal de ambos lóbulos, obteniendo un total de 8 cilindros. En este estudio la sextante clásica no diagnosticó un $20 \%$ de los tumores $^{44}$.

Biopsia de Saturación para biopsias repetidas

Stewart et al. ${ }^{48}$ introducen el concepto de biopsia de saturación en el año 2001. En 224 pacientes con biopsias previas negativas toma una media de 23 cilindros (14-45) bajo anestesia general, local o sedación y con un mapeo radial desde el cuerno lateral hacia la zona más medial $^{41}$. Obtienen una tasa de cáncer del 34\%, menor que el 38,4\% de tumores que encuentra Applewhite $^{49}$ en un estudio semejante utilizando la técnica de las cinco regiones.

Biopsia con toma sistemática de 12 cilindros

Singh et al. ${ }^{50}$ proponen esta estrategia, que consiste en la toma de los cilindros de la sextante clásica a los que añade tres muestras más de las zonas laterales, objetivando en su serie un $24 \%$ de tumores diagnosticados únicamente en las muestras laterales ${ }^{50}$.

\section{Biopsias transperineales 48,50}

Crawford et al. ${ }^{51}$ estudian sobre cadáveres y sobre piezas de prostatectomía radical un modelo de biopsia de próstata transperineal. Dividen la glándula en cuadrantes de 5 y 10 mm respetando la uretra y toman cilindros de cada cuadrante con una profundidad de $23 \mathrm{~mm}$. Encuentran una alta proporción de tumores clínicamente insignificantes sobre todo en la zona anterior (transicional), por otro lado encuentran una alta proporción de tumores clínicamente relevantes en la zona periférica posterior. Con esta técnica, sobre todo cuando se mapea la próstata en cuadrantes de 5 $\mathrm{mm}$, se obtienen valores de sensibilidad y de valor predictivo negativo muy altos para los tumores que en un futuro tendrían relevancia clínica. En otro estudio con esta modalidad, a 115 pacientes se les tomó una media de 18,4 cilindros con un rango entre 9-33, consiguiendo una tasa diagnóstica total del 43\%, si bien cuando se trataba de primeras biopsias la tasa ascendía al 49\% y cuando se trataba de biopsias de repetición sólo consiguieron una tasa del $29 \%{ }^{52}$.

Biopsia de próstata en función del volumen prostático y de la esperanza de vida

En 1997 surge la primera propuesta de individualizar el número de biopsias en función de la edad del paciente y del volumen prostático; Vashi et al. ${ }^{53}$, proponen un modelo matemático para determinar el número mínimo de cilindros necesario para identificar los tumores clínicamente relevantes. Teniendo en cuenta que el tiempo que tarda un cáncer de próstata en doblar su volumen es más o menos constante, (4-5 años para los T1-T2 $2^{54,55}$ ), y que las metástasis se correlacionan con el volumen del cáncer y con la supervivencia ${ }^{56}$. En los pacientes mayores (menor esperanza de vida) sólo será rentable detectar los tumores más grandes y por tanto será necesario un menor número de biopsias. De este modo elaboran unas tablas en las que lógicamente los pacientes más jóvenes (con mayor esperanza de vida) requerirán el mayor número de cilindros y los pacientes con menor esperanza de vida requerirán menor número de cilindros, oscilando el número definitivo en función del volumen prostático. Este modelo matemático llamado Nomograma de Viena (Tabla 1) ha sido validado por Remzi et al. ${ }^{57}$ en un estudio en el que comparan un grupo de 502 pacientes a los que biopsian con esta estrategia y otro grupo previo de 1051 pacientes al que biopsian con toma de ocho cilindros; el porcentaje de tumores diagnosticados con el nomograma es significativamente mayor que el de los pacientes a los realizan biopsia octante $(36,7 \% \text { vs } 20 \%)^{57}$. 
Tabla 1. Nomograma de Viena

\begin{tabular}{lcccc}
\hline $\begin{array}{l}\text { Volumen } \\
\text { prostático }\end{array}$ & $\mathbf{< 5 0 a}$ & $\begin{array}{c}\mathbf{5 1 - 6 0 a} \\
\text { Edad }\end{array}$ & $\mathbf{6 1 - 7 0 a}$ & $>\mathbf{7 0 a}$ \\
\hline $0-30$ & 8 & 8 & 8 & 6 \\
$31-40$ & 12 & 10 & 8 & 6 \\
$41-50$ & 14 & 12 & 10 & 8 \\
$51-60$ & 16 & 14 & 12 & 10 \\
$61-70$ & 18 & 16 & 14 & 12 \\
$>70$ & 18 & 18 & 16 & 14 \\
\hline
\end{tabular}

Importancia de la zona transicional en la biopsia de próstata

Un $20 \%$ de los tumores se originan en la zona transicional, pero el incluir en las primeras biopsias un cilindro de ésta, incrementa muy poco la rentabilidad diagnóstica $(1,8 \%-4,3 \%)^{58,59}$. Sin embargo en las segundas biopsias la rentabilidad aumenta hasta un $10 \%-13 \%{ }^{60,61}$. Lui et al. ${ }^{61}$ encuentran un 53\% de tumores en la zona transicional de segundas biopsias de pacientes con un PSA de $32 \mathrm{ng} / \mathrm{ml}$ y tacto rectal normal. Quizá por todo esto en las segundas biopsias esté justificada la toma de cilindros de esta zona. Para una correcta toma la aguja ha de sobrepasar la zona periférica, disparando entonces el resorte.

Biopsia de próstata en pacientes sin recto

En la bibliografía están descritas dos modalidades; biopsia de próstata transperineal guiada por ecografía transuretral ${ }^{62}$, y biopsia de próstata bajo control ecográfico directo transperineal ${ }^{63}$. Esta última modalidad tiene muy baja sensibilidad (10\%), y dada la dificultad técnica de la ecografia transuretral estos autores aconsejan un riguroso estudio de cáncer de próstata en aquellos pacientes a los que se les va a someter a una amputación abdominoperineal.

Algunos autores han estudiado la importancia de etiquetar separadamente los cilindros biopsiados con el fin de tener una información topográfica de la situación del tumor en la gándula ${ }^{64-67}$. Tombal et al. ${ }^{67}$ demuestran que el cáncer de próstata será organoconfinado cuando en la biopsia tres o menos cilindros son positivos para cáncer en un mismo lóbulo, el gleason es menor de 7 y el porcentaje de carga tumoral en la biopsia entre el $25-75 \%{ }^{67}$. Ravery et al. ${ }^{66}$ consideran que la escasa información que aporta la distribución topográfica del tumor en los cilindros se puede sustituir por el número de cilindros positivos y el porcentaje de carga tumoral.

En nuestro centro, realizábamos la biopsia de próstata transrectal ecodirigida con la técnica en sextante clásica más la obtención de uno-dos cilindros de las zonas hipoecoicas sospechosas en la escala de grises. Con esta estrategia obteníamos una tasa de detección de tumor del 26,7\% en pacientes con un PSA medio de 11,67 y volumen prostático medio de $52,6 \mathrm{ml}^{68}$. Con el fin de aumentar el rendimiento diagnóstico de las primeras biopsias se consensuó un nuevo protocolo en el que a pacientes a los que se les practica una primera biopsia se toman 10 cilindros, los sextantes más dos de cada cuerno lateral prostático. Cuando se trata de biopsias de repetición se indica una nueva estrategia con toma de 14 cilindros; los diez anteriores más dos cilindros de la zona transicional de cada lóbulo. En un trabajo mandado a prensa el pasado noviembre con una serie de 90 pacientes a los que se les sometió a una primera biopsia, con un PSA entre 4 y 10 , tacto rectal no sospechoso, los 4 cilindros "extra" de los cuernos laterales aumentaron el rendimiento diagnóstico un $27 \%$.

\section{COMPLICACIONES}

Cuando el urólogo indica una biopsia de próstata debe considerar los beneficios frente a los riesgos, y no solo los riesgos de la biopsia sino también los del tratamiento ulterior en el caso de detectar un tumor.

Hay descritas más de una centena de complicaciones menores, que incluyen, dolor, reacción vasovagal, rectorragia, hematuria ${ }^{67}$, etc.

El sangrado (rectorragia, hematuria o hemospermia) supone la complicación más frecuente de la biopsia de próstata transrectal. Las hematurias se dan según las series desde un 12,5 \%69 a un $80 \%{ }^{46}$. La incidencia de hemospermia oscila entre un $5,1^{70}$ y un $78,3 \%{ }^{71}$, mientras que la de rectorragias entre un $1,3 \%^{72}$ y un $58,6 \%{ }^{73}$. Las series son muy dispares y ésto se puede deber a la época en la que se realizó el estudio, al número de cilindros por biopsia, a la forma de valorar las complicaciones y al periodo que transcurre entre la biopsia y la evaluación. Además la mayo- 
ría de estas series están sujetas a sesgos del entrevistador, al sesgo anamnésico o al sesgo de atención de los pacientes que son advertidos de las complicaciones y se les entrega un cuestionario.

Naughton et al. ${ }^{74}$ correlacionan las tasas de sangrado con el número de cilindros y con la toma de biopsias más mediales; sin embargo Ghani et al. ${ }^{75}$ no encuentran dicha relación pues objetivan una incidencia de hematuria macroscópica de $44 \%, 41 \%$ y $39 \%$ en los pacientes sometidos a biopsia con toma de 6,8 ó 12 cilindros respectivamente, la incidencia de hemospermia fue del 13\%, $16 \%$ y $12 \%$ y de rectorragia del $17 \%, 26 \%$ y $27 \%$. En dicho estudio la rectorragia era más frecuente en los pacientes sometidos a biopsias ampliadas. En este estudio no encuentran diferencias en cuanto a la severidad o duración del sangrado. En todos los casos fue leve y autolimitada.

Hay descritos casos anecdóticos de hemorragias importantes como la descrita por Kaneko ${ }^{76}$ de un paciente que desarrolló un hematoma masivo retroperitoneal por lesión de la arteria prostática derecha y que requirió embolización arterial para el tratamiento. También hay otro caso de hematuria que requirió coagulación endoscópica ${ }^{77}$.

Las rectorragias intraoperatorias suelen ser de escasa importancia y basta con un taponamiento durante unos minutos con el propio transductor o con una compresa lubricada.

De un $0,2 \% 78$ a un $10 \%{ }^{79}$ sufren retención aguda de orina sobre todo en las biopsias con toma de más de 12 cilindros.

Gracias a la profilaxis antibiótica las complicaciones infecciosas son escasas y la mayoría de los casos se pueden tratar de manera ambulatoria. En una serie española encuentran que el $11,5 \%$ de sus pacientes presentan fiebre post-biopsia, y el $1,9 \%$ tuvo que permanecer en el hospital más de 24 horas $^{80}$. Se encuentran en la literatura algunos casos con complicaciones mayores como una meningitis bacteriana causada por $E$. coli multirresistente en un paciente al que se le practica una biopsia prostática previa profilaxis con quinolona ${ }^{81}$.
Tampoco se han encontrado diferencias en cuanto a la tasa de complicaciones cuando se compararon las biopsias transperineales con las transrectales ${ }^{82}$.

\section{DISCUSIÓN}

Actualmente, pese al importante desarrollo de las técnicas de imagen, y el avance en los marcadores tumorales, la biopsia de próstata sigue siendo la prueba ineludible para el diagnóstico definitivo del cáncer de próstata.

El número y distribución de los cilindros es difícil de determinar. No es factible un metaanálisis riguroso entre los distintos estudios pues las características de los pacientes no son equiparables. La tasa de detección variará dependiendo del número de biopsias, la localización de las mismas, de que se trate de pacientes de screening o pacientes con sospecha de cáncer prostático por aumento del PSA o por tacto rectal sospechoso (Tabla 2). Deducimos por tanto que no existe por el momento una estrategia ideal.

Numerosos estudios indican que el aumento del número de cilindros aumenta la tasa de detección de cáncer ${ }^{44-48,53,83}$. El mero hecho de lateralizar las punciones de las biopsias en sextante aumenta un $23 \%$ la tasa de diagnóstico de cáncer ${ }^{12,83}$. También ha quedado reflejado que el aumentar el numero de cilindros a 10, 12 ó 13 cilindros aumenta la proporción de diagnósticos en un 25,5\%, 22\%, y 35\% respectivamente $26,84,85$. No obstante, otros trabajos muestran resultados contrarios ${ }^{74,86,87}$.
Tabla 2

\begin{tabular}{lclcc}
\hline Técnica & No Cilin & Serie & N & Tasa diagnóstica \\
\hline Sextante Clásica & 6 & H. La Fe & 303 & $26,7 \% 68$ \\
10 cilindros & 10 & H. La Fe & 90 & $41 \% 90$ \\
Cinco regiones & 13 & Eskew & 119 & $40,3 \% 46$ \\
Doble sextante & 12 & Applewhite* & 125 & $38,4 \% 49$ \\
11 cilindros & 11 & Bevine & 137 & $31 \% 47$ \\
Ocho cilindros & 8 & Presti & 483 & $42 \% 44$ \\
Saturación & $14-45$ & Stewart* & 224 & $34 \% 48$ \\
Sistemática 12 cil & 12 & Elabbady & 176 & $36,4 \% 91$ \\
Transperineal & 12 & Takenaka & 247 & $39,7 \% 92$ \\
Nomograma Viena & & Remzi & 502 & $36,7 \% 57$ \\
\hline
\end{tabular}

* series de biopsias de repetición 
El hecho de que en la literatura existan tasas de detección de cáncer del 37\% en biopsias de repetición traduce que las biopsias previas habían dejado escapar muchos tumores, y por lo que se deduce que la estrategia no era la idónea.

La trascendencia clínica de los tumores "extra" diagnosticados por las biopsias ampliadas es un tema controvertido. Existe un temor a la detección de tumores clínicamente irrelevantes y al consiguiente sobretratamiento del cáncer de próstata. Hasta la fecha Eskew et al. ${ }^{46}$ así como Presti et al. ${ }^{44}$, han demostrado en sus estudios que el aumentar el número de muestras de las biopsias no aumenta de forma significativa el porcentaje de tumores insignificantes clinicamente, sino que detecta tumores en un estadio más precoz.

La morbilidad asociada a la biopsia prostática es tolerable, y las tasas de complicaciones mayores que requieren hospitalización son bajas y oscilan entre el $0 \%^{88}$ y el $4 \%^{89}$. Se trata de una técnica que si bien es incómoda para el paciente, es lo suficientemente segura como para no obviar su repetición en caso necesario.

\section{CONCLUSIONES}

La biopsia de próstata transrectal ecodirigida es la técnica de elección para el diagnóstico del cáncer de próstata.

Para efectuar la biopsia de próstata es necesario controlar el dolor; una cobertura antibiótica de amplio espectro, y a nuestro juicio y a falta de estudios de mayor envergadura por el momento consideramos recomendable cierta preparación del recto previa. Hasta hoy no tenemos evidencia clara de que el aumento del número de cilindros en las muestras incremente de manera significativa la morbilidad de la prueba.

Se deduce de los resultados de las series más importantes que para aumentar la rentabilidad de la prueba es necesaria aumentar de forma moderada el número de tomas, sobre todo a expensas de las zonas laterales de la próstata.

Por tanto, a nuestro juicio son válidas las estrategias que contengan un mínimo de cuatro cilindros de las zonas más lateroprostáticas, reservando las biopsias de saturación con toma de cilindros de la zona transicional para pacientes a los que se le va a someter a una biopsia de repetición por persistencia de una alta sospecha de cáncer prostático.

\section{REFERENCIAS}

1. Terris MK. Prostate biopsy strategies: past, present, and future. Urol Clin N Am. 2002;29(1):205-212.

2. Schlegel JU, Diggdon P, Cuellar J. The use of ultrasound for localizang renal calculi. J. Urol. 1961;86;367-369.

3. Watanabe $H$, Kato $H$, Kato $T$, Morita $M$, Tanaka $M$. Diagnostic aplication of the ultrasoundotomography for the prostate. Nippon Hinyokika Gakkai Zasshi. 1968;59 (4):273-279.

4. Lee F, Gray JM, McLeary RD, Meadows TR, Kumasaka GH, Borlaza GS, et al. Transrectal ultrasound in the diagnosis of prostate cancer: location, echogenicity, histopathology, and staging. Prostate. 1985;7(2):117-129.

5. Radge H, Aldape HC, Bagley CM Jr. Ultrasound guided prostate biopsy: biopsy gun superior to aspiration. Urology. 1988;32(6):503-506.

6. Lee F, Torp-Pedersen ST, Siders DB. The role of transrectal ultrasound in the early detection of prostate cancer. CA Cancer J Clin. 1989;39(6):337-360.

7. Hodge KK, Macneal JE, Stamey TA. Ultrasound guided transrectal core biopsy of the palpably abnormal prostate. J Urol. 1989;142(1):66-70.

8. Carter HB, Hamper UM, Sheth S, Sanders RC, Epstein JI, Walsh PC. Evaluation of ultrasound in the early detection of prostate cancer. J Urol. 1989;142(4):1008-1010.

9. Ellis WJ, Chetner MP, Preston SD, Brawer MK. Diagnosis of prostatic carcinoma: The yield of serum prostate specific antigen, digital rectal examination and transrectal ultrasonography. J Uro 1994 Nov; 152(5 Pt 1):1520-1525.

10. Hammerer PG. Sonographic diagnosis of prostatic cancer. Radiologe. 1994;34(3):116-121.

11. Hammerer PG, Huland H. Systematic sextant biopsies in 651 patients referred for prostate evaluation. J Urol. 1994;151(1):99-102.

12. Rodríguez-Patrón Rodríguez R, Mayayo Dehesa T, Burgos Revilla FJ, Alonso González M, Lennie Zucharino A, García González R. Resultados de una serie de 6000 pacientes con biopsia transrectal ecodirigida de próstata. Parte I; Anatomía Patológica, Tacto rectal, Ecografía transrectal y PSA. Arch Esp Urol. 2005;58(7):623-634.

13. Pelzer A, Bektic J, Berger AP, Pallwein L, Halpern EJ, Horninger W, Bartsch G, Frauscher F. Prostate cancer detection in men with prostate specific antigen 4 to 10 $\mathrm{ng} / \mathrm{ml}$ using a combined approach of contrast enhanced color Doppler targeted and systematic biopsy. J Urol. 2005; 173(6): 1926-1929.

14. Frauscher F, Pallwein L, Klauser A, Berger AP, Koppelstaetter F, Gradl J et al. Ultrasound contrast agents and prostate cancer Radiologe. 2005;45(6):544-551.

15. Mehta SS, Azzouzi AR, Hamdy FC. Three dimensional ultrasound and prostate cancer. World J Urol. 2004;22(5): 339-345.

16. Ito H, Kamoi K, Yokoyama K, Yamada K, Nishimura T. Visualization of prostate cancer using dynamic contrastenhanced MRI: comparison with transrectal power Doppler ultrasound. Br J Radiol. 2003;76(909):617-624.

17. Yuen JS. Re Comparison of contrast enhanced color Doppler targeted biopsy with conventional systematic biopsy: impact on prostate cancer detection. J Urol. 2003; 170(1):192-193.

18. Carter B, Partin AW. Urology Campbell 8Ed 816-817.

19. Vallancien G, Prapotnich D, Veillon B. et al. Systemic prostatic biopsies in 100 men with no suspicion of cancer on digital rectal examination. J Urol. 1991;146(5):1308-1312. 
20. Brown RW, Warner JJ. Turner BI et al. Bacteremia and bacteriuria after transrectal prostatic biopsy. Urology. 1981;18(2): 145-148

21. Lindert KA, Kabalin JN, Terris MK. Bacteremia and bacteriuria after transrectal ultrasound guided prostate biopsy. J Urol. 2000;164(1):76-80.

22. Carey JM, korman HJ. Transrectal ultrasound guided biopsy of the prostate. do enemas decrease clinically significant complications? J Urol. 2001;166(1):82-85.

23. Davis M, Sofer M, Kim SS, Soloway MS. The procedure of transrectal ultrasound guided biopsy of the prostate: a survey of patient preparation and biopsy technique. $\mathrm{J}$ Urol. 2002; 167(2Pt1):566-570.

24. Clements R, Aideyan OU, Griffiths GJ Peeling WB. Side effects and patient acceptability of transrectal biopsy of the prostate. Clin Radiol. 1993;47(2):125-126.

25. Collins GN, Lloyd SN, Hehir M, McKelvie GB. Multiple transrectal ultrasound-guided prostatic biopsies true morbidity and patient acceptance. Br J Urol. 1993;71(4):460463.

26. Nash PA, Bruce JE, Indudhara R, Shinohara K. Transrectal ultrasound guided prostatic nerve blockade eases systematic needle biopsy of the prostate. J Urol. 1996;155 (2):607-609.

27. Soloway M, Obek C. Periprostatic local anesthesia before ultrasound guided prostate biopsy. J Urol. 2000;163(1): 172-173.

28. Wu CL, Carter BH, Naqibudin M et al. Effect of local anesthesics on patient recovery after transrectal biopsy. Urology 2001;57(5):925-959.

29. Taverna G, Maffezini M, Benetti A, Seveso M, Giusti G, Graziotti P. A single injection of lidocaine as local anaesthesia for ultrasound guided biopsy of the prostate. J Urol. 2002;167(1):222-223.

30. Seymour H, Perry MJA, Lee-Elliot C. Pain after transrectal ultrasonographi-guided biopsy. The adventages of periprostatic local anaesthesia. BJU Int. 2001;88(6):540-544.

31. Issa MM, Bux S, Chun T, Petros JA, Labadia AJ, Anastasia $\mathrm{K}$ et al. A randomized prospective trial of intrarectal lidocaine for pain control during transrectal prostate biopsy: the Emory University experience. J Urol. 2000;164(2):397-399.

32. Alavi AS, Soloway MS, Vaidya A, Lynne CM, Gheiler EL. Local anesthesia for ultrasound guided prostate biopsy: a prospective randomized trial comparing 2 methods. J Urol. 2001;166:1343-1345.

33. Massod J, Shah N, Lane T. Nitrous Oxide (entonox) inhalation and tolerance of transrectal ultrasound guided prostate biopsy: a double-blind randomiced controlled study. J Urol. 2002;168(1): 116-120.

34. Peters JL, Thompson AC, Mcnicholas TA et al. Increased patient satisfaction form transrectal ultrasound-guided biopsy under sedation BJU Int. 2001;87(9):827-830.

35. Moinzadeh A, Mourtzinos A, Triaca V. A randomised double blind prospective study evaluating patient tolerance of transrectal ultrasound-guided biopsy of the prostate using prebiopsy rofecoxib. Urology 2003;62(6):1054-1057.

36. Haq A, Patel HRH, Habib Mr. Diclofenac suppository analgesia for transrectal ultrasound guided biopsy of the prostate: a double blind, rendomized controlled trial. J Urol. 2004;171(4):1489-1491.

37. Bastide C, Lechevallier E, Eghazarian C, Ortega JC, Coulange C. Tolerance of pain during transrectal ultrasound-guided biosy of the prostate: risk factors. Prostate Cancer Prostatic Dis. 2003;6(3):239-241.
38. Griffith BC, Morey AF, Ali-Khan MM, Canby-Hagino E, Foley JP, Rozanski TA. Single dose levofloxacin prophylaxis for prostate biopsy in patients at low risk. J Urol. 2002; 168(3):1021-1023.

39. Bonillo MA, Bosquet M, Arlandis S, Trassierra M, Ramírez M, Jiménez JF. Profilaxis antibiótica en la biopsia de prostate. C-59 LXX Congreso Nacional de Urología Jun 2005.

40. Sieber PR, Rommel FM, Agusta VE. Antibiotic prophylaxis in ultrasound guided transrectal prostate biopsy. J Urol. 1997;157(6):2199-2200.

41. Aus G, Ahlgren G, Bergdahl S. Infection after transrectal core biopsies of the prostate: risk factors and antibiotic prophylaxis. Br J Urol. 1996;77(6):851-855.

42. Norberg M, Holmberg L, Haggman M. Determinants of complications after multiple transrectal core biopsies of the prostate. Eur Radiol. 1996;6(4):457-459.

43. Stamey TA. Making the most out of six systematic sextant biopsies. Urology 1995;45(1):2-12.

44. Presti JC Jr, Chang JJ, Bhargava V, Shinohara K. The optimal systematic prostate biopsy scheme should include 8 rather than 6 biopsies: results of a prospective clinical trial. J Urol. 2003;163(1):163-166.

45. Babaian RJ, Toi A, Kamoi K, Troncoso P, Sweet J, Evans R et al. A comparative analysis of sextant and an extended 11-core multisite directed biopsy strategy. J Urol. 2000; 163(1):152-157.

46. Eskew LA, Bare RL, McCullough DL. Systematic 5 region prostate biopsy is superior to sextant method for diagnosing carcinoma of the prostate. J Urol. 1997;157(1):199202.

47. Levine MA, Ittman M, Melamed J, Lepor H. Two consecutive sets of transrectal ultrasound guided sextant biopsies of the prostate for the detection of prostate cancer. J Urol.1998;159(2):471-475.

48. Stewart CS, Leibovich BC, Weaver AL, Lieber MM. Prostate cancer diagnosis using a saturation needle biopsy technique after previous negative sextant biopsies. J Urol. 2001;166(1):86-91

49. Applewhite JC, Matlaga BR, McCullough DL. Results of the 5 region prostate biopsy method: the repeat biopsy population. J Urol. 2002;168(2):500-503.

50. Singh H, Canto EI, Shariat SF, Kadmon D, Miles BJ, Wheeler TM et al. Improved detection of clinically significant, curable prostate cancer with systematic 12-core biopsy. J Urol. 2004;171(3):1089-1092.

51. Crawford ED, Shandra S, Wilson SS, Torkko KC, Hirano D, Stewart IS et al. Clinical staging of prostate cancer: a computer-simulated study of transperineal Prostate biopsy. Br Jour Urol. 2005;96(7):999-1004.

52. Furuno T, Demura T, Kaneta T, Gotoda H, Muraoka S, Sato $\mathrm{T}$ et al. Difference of cancer core distribution between first and repeat biopsy: in patients diagnosed by extensive transperineal ultrasound guided template prostate biopsy. Prostate 2004;58(1):76-81.

53. Vashi AR, Wojno KJ, Gillespie B, Oesterling JE. A model for the number of cores per prostate biopsy based on patient age and prostate gland volume. J Urol. 1998;159(3):920924.

54. Schmid H, McNeal JE, Stamey TA. Observations on the doubling time of prostate cancer: the use of serial prostate-specific antigen in patients with untreated disease as a measure of increasing cancer volume. Cancer 1993;71(6): 2031-2040. 
55. Dugan JA, Bostwick DG, Myers RP, Qian J, Bergstralh EJ, Oesterling JE. The definition and preoperative prediction of clinically insignificant prostate cancer. JAMA. 1996;275(4):288-294.

56. Bostwick DG, Graham SD Jr, Napalkov P, Abrahamsson PA, di Sant'agnese PA, Algabe F et al. Staging of early prostate cancer: a proposed tumor volume-based prognostic index. Urology. 1993;41(5):403-411.

57. Remzi M, Fong YK, Dobrovits M, Anagnostou T, Seitz C, Waldert $M$ et al. The vienna nomogram: Validation of a novel biopsy strategy defining the optimal number of cores based on patient age and total prostate volume. J Urol. 2005; 174(4Pt1):1256-1261.

58. Bazinet M, Karakiewicz PI, Aprikian AG, Trudel C, Aronson $\mathrm{S}$, Nachabe $\mathrm{M}$ et al. Value of systematic transition zone biopsies in the early detection of prostate cancer. J Urol. 1996;155(2):605-606.

59. Epstein JI, Walsh PC, Sauvegeot J, Carter HB. Use of repeat sextant and transition zone biopsies for assessing extent of prostate cancer. J Urol. 1997;158(5):1886-1890.

60. Keetch DW, Catalona WJ. Prostatic transition zone biopsies in men with previous negative biopsies and persistently elevated serum prostatic specific antigen values. J Urol. 1995;154(5):1795-1797.

61. Lui PD, Terris MK, McNeal JE, Stamey TA. Indications for ultrasound guided transition zone biopsies in the detection of prostate cancer. J Urol. 1995;153(3Pt2):1000-1003.

62. Seaman EK, Sawczuk IS, Fatal M, Olsson CA, Shabsigh R. Transperineal prostate needle biopsy guided by transurethral ultrasound in patients without a rectum. Urology. 1996;47(3):353-355.

63. Shinghal R, Terris MK. Limitations of transperineal ultrasound-guided prostate biopsies. Urology. 1999;54(4):706708.

64. Taneja SS, Penson DF, Epelbaum A, Handler T, Lepor H. Does site specific labeling of sextant biopsy cores predict the site of extracapsular extension in radical prostatectomy surgical specimen. J Urol. 1999;162(4):1352-1357.

65. Sanwick JM, Dalkin BL, Nagle RB. Accuracy of prostate needle biopsy in predicting extracapsular tumor extension at radical retropubic prostatectomy: application in selecting patients for nerve-sparing surgery. Urology. 1998;52:814-818.

66. Ravery V, Boccon-Gibod LA, Dauge-Geffroy MC et al Systematic biopsies accurately predict extracapsular extension of prostate cancer and persistent/recurrent detectable PSA after radical prostatectomy. Urology. 1994;44(3):371-376.

67. Tombal B, Tajeddine N, Cosyns, Feyaerts A, Opsomer R, Wese $\mathrm{F}$ et al. Does site-specific labelling and individual processing of sextant biopsies improve the accuracy of prostate biopsy in predicting pathological stage in patients with T1c prostate cancer? Br Jour Urol. 2002;89(6):543-548.

68. Gómez Ferrer A. Análisis computerizado de la imagen computerizada transrectal en el cáncer de próstata localizado. 2004 Tesis Doctoral Univ. Valencia.

69. Aus G, Hermansson CG, Hugosson J, Pedersen KV. Transrectal ultrasound examination of the prostate: complications and acceptance by patients. $\mathrm{Br} \mathrm{J}$ Urol. 1993;71(4):457-459.

70. Torp-Pedersen S, Lee F, Littrup PJ, Siders DB, Kumasaka $\mathrm{GH}$, Solomon MH et al. Transrectal biopsy of the prostate guided with transrectal US. longitudinal and multiplanar scanning. Radiology 1989;170(1Pt1):23-27.
71. Peyromaure M, Ravery V, Messas A, Toublanc M, BocconGibod L, Boccon-Gibod L. pain and morbidity of an extensive prostate 10- biopsy protocol: a prospective study in 289 patients. J Urol. 2002;167(1):218-221.

72. Raaijmakers R, Kirkels WJ, Roobol MJ, Wildhagen MF, Schroder FG. Complication rates and risk factors of 5802 transrectal ultrasound guided sextant biopsies of the prostate within a population based screening program. Urology. 2002;60(5):826-830.

73. Makinen T, Auvinen A, Hakama M, Stenman UH, Tammela TL. Acceptability and complications of prostate biopsy in population-based SA screening versus routine clinical practice: a prospective, controlled study. Urology 2002;60 (5):846-850.

74. Naughton CK, Ornstein DK, Smith DS, Catalona WJ. Pain and morbidity of transrectal ultrasound guided prostate biopsy: a prospective randomized trial of 6 versus 12 cores. J Urol. 2000;163(1):168-171.

75. Ghani KR, Dundas D, Patel U. Bleeding after transrectal ultrasonography-guided prostate biopsy: a study of 7-day morbidity after a six-, eight- and 12-core biopsy protocol. BJU Int. 2004;94(7):1014-1020.

76. Kaneko T, Suzuki T, Matsushita N, Yoshida I. Transcatheter arterial embolization for bleeding of prostatic artery after prostate biopsy Nippon Hinyokika Gakkai Zasshi. 2003;94(7):693-695.

77. Brullet E, Guevara MC, Campo R, Falcó J, Puig J, Prera A et al: Massive rectal bleeding following transrectal ultrasound-guided prostate biopsy. Endoscopy. 2000;32(10): 792-796.

78. Enlund AL, Varenhorst E. Morbidity of ultrasound-guided transrectal core biopsy of the prostate without prophylactic antibiotic therapy. A prospective study in 415 cases. $\mathrm{Br}$ J Urol. 1997;79(5):777-780.

79. Borboroglu PG, Comer SW, Riffenburgh RH, Amling CL. Extensive repeat transrectal ultrasound guided prostate biopsy in patients with previous benign sextant biopsies. $\mathrm{J}$ Urol. 2000;163(1):158-162.

80. Lujan Galan M, Paez Borda A, Fernandez Gonzalez I, Romero Cajigal I, Gomez de Vicente JM, Berenguer Sanchez A. Efectos adversos de la biopsia prostática transrectal. Un análisis de 303 procedimientos. Actas Urol Esp. 2001;25(1):46-49.

81. Rodriguez-Patron Rodriguez R, Navas Elorza E, Quereda Rodriguez-Navarro C, Mayayo Dehesa T. Meningitis caused by multiresistant E. coli after an echo-directed transrectal biopsy Actas Urol Esp. 2003;27(4):305-307.

82. Miller J, Perumalla C, Heap G. Complications of transrectal versus transperineal prostate biopsy. ANZ J Surg. 2005;75(1-2):48-50.

83. Bauer JJ, Zeng J, Weir J, Zhane W, Sesterhenn IA, Connelly RR et al. Three-dimensional computer-simulated prostate models: lateral prostate biopsies increase the detection rate of prostate cancer. Urology. 1999;53(5):961967.

84. Eskicorapci SY, Bayda DE, Akbal C, Sofikerim M, Gunay M, Ekici S et al. An extended 10-core transrectal ultrasonography-guided prostate biopsy protocol improves the detection of prostate cancer. Eur Urol. 2004;45(4):444448.

85. Beurton D, Barthe'le'my Y, Fontaine E. Twelve systematic prostate biopsies are superior to sextant biopsies for diagnosing carcinoma: a prospective randomized study. $\mathrm{Br} \mathrm{J}$ Urol. 1997;80:239-242. 
86. Naughton CK, Miller DC, Mager DE, Ornstein DK, Catalona WJ. A prospective randomized trial comparing 6 versus 12 prostate biopsy cores: impact on cancer detection. J Urol. 2000;164(2):388-392.

87. Rodríguez-Patrón Rodríguez R, Mayayo Dehesa T, Alonso González M, Burgos Revilla FJ, García González R, Lennie Zucharino A. Rentabilidad de la biopsia de prostate ampliada con 10muestras prostáticas. Estudio prospectivo sobre 222 pacientes. Arch Esp Urol. 2006;59(1):15-24.

88. Torp-Pedersen S, Lee F, Littrup PJ, Siders DB, Kumasaka $\mathrm{GH}$, Solomon $\mathrm{MH}$ et al. Transrectal biopsy of the prostate guided with transrectal US. longitudinal and multiplanar scanning. Radiology 1989;170(1Pt1):23-27.

89. Peters JL, Thompson AC, McNicholas TA, Hines JE, Hanbury DC, Boustead GB. Increased patient satisfaction from transrectal ultrasonography and biopsy under sedation. BJU Int. 2001;87(9):827-830.

90. Ramírez M, Trasierra M, Delgado F, Gómez L, Arlandis S, Pontones JL, Jiménez JF. Estudio comparativo entre la biopsia sextante y la ampliada con toma de 10 cilindros. Póster. Reunión Nacional grupo Uro-Oncológico. 2006. Granada.

91. Elabbady AA, Khedr MM. Extended 12-core prostate biopsy increases both the detection of prostate cancer and the accuracy of gleason score. Eur Urol. 2006;49(1):49-53.

92. Takenaka A, Hara R, Hyodo Y, Ishimura T, Sakai Y, Fujioka $\mathrm{H}$ et al. Transperineal extended biopsy improves the clinically ignificant prostate cancer detection rate: A comparative study of 6 and 12 biopsy cores. Int $\mathrm{J}$ Urol. 2006;13(1):10-14.

Correspondencia autor: Dr. M. Ramírez Backhaus Servicio de Urología. Hospital Universitario La Fe Avda. Campanar, 21 - 46009 Valencia

Tel.: 963862700

E-mail autor: ramirezbackhaus@yahoo.es Información artículo: Revisión - Cáncer de próstata Trabajo recibido: enero 2007

Trabajo aceptado: abril 2007 